EDITORIAL

\title{
The debate on public place defibrillators: charged but shockingly ill informed
}

J P Pell

Heart 2003;89:1375-1376

There is growing interest in developing strategies which will enable defibrillation to be administered while waiting for the emergency medical service to arrive

$\mathrm{N}$ early half of all deaths from cardiovascular disease are sudden and unexpected. ${ }^{1}$ Most sudden cardiac deaths occur before hospital admission. The delay from cardiopulmonary arrest to defibrillation is a significant independent predictor of survival. ${ }^{2}$ Two minutes after the arrest around two thirds of victims have rhythms amenable to defibrillation, but this percentage decreases rapidly with time. Obviously, it is imperative to minimise the delay from calling the emergency medical service to their arrival at the arrest scene. However, some delay is inevitable. Therefore, interest has grown in complementary strategies whereby defibrillation can be administered while waiting for the emergency medical service to arrive.

Individuals known to be at very high risk of arrest can be provided with implantable cardioverter-defibrillators or automated external defibrillators for their personal use. However, such individuals account for a small percentage of all pre-hospital arrests. ${ }^{3}$ Therefore, such strategies have relatively little impact at a population level. The American Heart Association has suggested a number of possible population strategies. ${ }^{4}$ Firstly traditional first responders, such as police and fire personnel, can be provided with automated external defibrillators and training in their use. Secondly, novel first responders can be identified and trained. One example includes the provision of defibrillators on aircraft for use by trained airline personnel. Thirdly, training can be given to groups of lay volunteers. Finally, it is possible to adopt an alternative approach by locating "intelligent" defibrillators in public places for use by lay bystanders with minimal or no training in their use. This approach is analogous to that adopted for fire extinguishers.

\section{LOCATING DEFIBRILLATORS IN PUBLIC PLACES}

Locating defibrillators in public places, such as airports, is associated with a high survival among individuals on whom they are used. For example, 70 automated defibrillators were located in three airports in Chicago, USA. Over the subsequent two years, 18 people were shocked for ventricular fibrillation (VF) and 10 (56\%) survived. ${ }^{5}$ Unfortunately this study was typical of many in that the investigators were unable to provide meaningful comparative data. They compared their survival to the $2.9 \%$ survival reported in an 11 year old study. ${ }^{6}$ This very low figure was based on all arrests, rather than just VF arrests, and all locations, rather than just public places or airports. Therefore, the comparison was inappropriate. The choice of comparison group is critical if bias is to be avoided. Cardiac arrests that occur in public places are significantly more likely to be witnessed and, therefore, already have the shortest ambulance delays. Among arrests in public places, $12.8 \%$ are attended within three minutes compared with only 9.7\% in more secluded locations. ${ }^{7}$ As a result, people who arrest in public places are already more likely to be shocked (72.9\% v 56.2\%) and, therefore, have the highest baseline survival $(8.7 \% \quad v 4.2 \%){ }^{7}$ People with the foresight to arrest in airports are more fortunate still since half of the "untrained bystanders" in Chicago airports had medical degrees!

As with implantable defibrillators, public place defibrillators may have a limited impact at the population level. The vast majority of prehospital arrests occur in places completely unsuitable for locating public place defibrillators such as private homes and open countryside. ${ }^{7}$ Studies have consistently shown that only 16$18 \%$ of pre-hospital arrests occur in public places, ${ }^{7-9}$ and less than $2 \%$ of pre-hospital arrests occur in public places in which the frequency of arrests exceeds one every 10 years. ${ }^{8}$ Using data from the Heartstart Registry we were able to demonstrate that making defibrillators as ubiquitous as fire extinguishers would improve overall survival from non-crew witnessed arrests from $5.0 \%$ to only $6.5 \%{ }^{7}$ By contrast, providing defibrillators to the fire service, which already has a five minute response time, could potentially double overall survival. ${ }^{2}$

\section{COST EFFECTIVENESS}

Public place defibrillators are only one option among a number of potential strategies aimed at reducing time to defibrillation. Therefore, it is imperative to consider not only their clinical effectiveness but also their cost effectiveness. A few studies have attempted to calculate the cost effectiveness of public place defibrillators. However, all have been limited by a lack of data on either benefit or cost. Caffrey and colleagues used the data from the Chicago airports to calculate a cost per life gained. ${ }^{5}$ However, as already discussed, the increase in survival was exaggerated greatly by the choice of an inappropriate comparison group. In England, the Department of Health has committed more than 
$£ 3$ million (€4.3 million) to provide defibrillators in 72 public places. Woollard attempted to model the cost effectiveness of this strategy. ${ }^{10}$ However, a crucial assumption in his model was that $50 \%$ of all future public place arrests in the whole of England will occur in the 72 sites in which public place defibrillators will be located. The published evidence does not support this assumption. ${ }^{7-9}$

In the cost effectiveness model of public place defibrillators conducted by Nichol and colleagues, they assumed that the "density of distribution of defibrillators in a community was such that one device was available for each cardiopulmonary arrest that occurred in public". ${ }^{11}$ However, they acknowledged having no data on the costs needed to achieve this level of coverage. They included an additional cost per arrest of only US\$1200 in their public place defibrillator model compared with an additional cost per arrest of $\$ 3300$ in a separate police first responder model. Only $\$ 300$ of the police cost was attributed to training. Hence, after subtracting the costs of training, the additional cost per arrest used in the police model was still 2.5 times higher than that used in the public place defibrillator model.

The location of future arrests cannot be predicted accurately. Public place defibrillators are frequently carried on foot and can, therefore, cover only a small geographical area. By contrast, police vehicles can transport defibrillators rapidly across a much larger geographical area. Hence, public place defibrillators have to be distributed more densely to achieve the same level of coverage as a dynamically deployed system. Therefore, it is difficult to conceive how the cost per arrest of providing "one device...for each cardiac arrest that occurred in public" could be achieved more cheaply using public place defibrillators than a police first responder model.

\section{INADEQUATE INFORMATION}

The American Heart Association and the Department of Health in England are both strong advocates of public place defibrillators. Public place defibrillators are being provided in increasing numbers in both countries. ${ }^{412}$ However, decisions to expand provision of public place defibrillators have been taken in advance of adequate information on their clinical and cost effectiveness. Published data on clinical effectiveness are based entirely on observational studies, many of which have lacked adequate baseline or comparative data. As yet, there are no robust data on cost effectiveness or cost utility. Without information on the value for money of such a strategy, it is impossible to determine whether greater improvements in survival and quality of life could be achieved by investing the same amount of money in alternative strategies such as reduced emergency medical service response times or the provision of defibrillators to other first responders.

The decision on whether or not to provide public place defibrillators should be based on objective evidence and not political expediency. Additional resources provided to existing services often go unnoticed by the public and media. Providing defibrillators to other first responders, such as the fire service, is a sensitive and, therefore, politically high risk strategy. By contrast, public place defibrillators are a "good news story". They are novel, highly visible, and empower the public and, therefore, attract positive media coverage. These factors in isolation are not sufficient to justify their funding from public monies. They need to be supported by clinical and economic evidence.

Five years ago, the American Heart Association highlighted the need for clinical trials of public place defibrillators, and recommended that these be undertaken before public place defibrillators become more widespread. ${ }^{4}$ Randomised trials are only now being conducted. Clinical and economic evidence is required from randomised controlled trials before further expansion of public place defibrillators can be justified.

\section{REFERENCES}

1 Zipes DP, Wellens HJ. Sudden cardiac death. Circulation 1998:98:2334-51.

2 Pell JP, Sirel JM, Marsden AK, et al. Potential impact of reducing ambulance response times on deaths from out of hospital cardiac arrest: a prospective cohort study. BMJ 2001;322:1385-8.

3 Myerburg RJ, Kessler KM, Castellanos A. Sudden cardiac death: structure, function and time-dependence of risk. Circulation 1992;85(suppl I):I2-I10.

4 Nichol G, Hallstrom AP, Kerber R, et al. American Heart Association report on the second public access defibrillation conference, April 17-19, 1997. Circulation 1998;97:1309-14.

5 Caffrey SL, Willoughby PJ, Pepe PE, et al. Public use of automated external defibrillators. N Engl J Med 2002;347:1242-7.

6 Becker LB, Ostrander MP, Barrett J, et al. Outcome of CPR in a large metropolitan area - where are the survivors? Ann Emerg Med 1991;20:355-61.

7 Pell JP, Sirel JM, Marsden AK, et al. Potential impact of public access defibrillators on overall survival following out of hospital cardiopulmonary arrest. BMJ 2002;325:515-7.

8 Becker L, Eisenberg M, Fahrenbruch C, et al. Public locations of cardiac arrest. Implications for public access defibrillation. Circulation 1998;97:2106-9.

9 Gratton M, Lindholm DJ, Campbell JP. Public access defibrillators. Prehospital emergency care 1999;3:303-5.

10 Woollard M. Public access defibrillators: a shocking idea? J Public Health Med 2001;23:98-102.

11 Nichol G, Hallstrom AP, Ornato JP, et al. Potential cost-effectiveness of public access defibrillation in the United States. Circulation 1998;97:1315-20.

12 Davies CS, Colquhoun M, Graham S, et al. Defibrillators in public places: the introduction of a national scheme for public access defibrillation in England. Resuscitation 2002;52:13-21.

\section{ELECTRONIC PAGES}

\section{Heart Online case reports: www.heartjinl.com}

$\mathrm{T}$ he follow electronic only article is published in conjunction with this issue of Heart.

\section{Single coronary artery: a familial clustering}

\section{P G Horan, G Murtagh, P P McKeown}

A 59 year old man undergoing investigation for chest pain was found at elective coronary angiography to have a single coronary artery; the left coronary had a normal distribution, with the right coronary originating as a continuation of the atrioventricular circumflex. His 30 year old daughter was admitted for elective coronary angiography for further investigation of a dilated cardiomyopathy. She was also found to have a single coronary artery. However, in her case, the right and left coronary arteries arose from the right sinus of Valsalva; the right coronary had a normal distribution, the left coronary passed anterior to the pulmonary trunk and aorta.

(Heart 2003;89:e27) www.heartjnl.com/cgi/content/full/89/ 12/e27 